\title{
Mechanisms of reduced implant stability in osteoporotic bone
}

\section{Journal Article}

Author(s):

Ruffoni, D.; Muller, R.; van Lenthe, G. Harry

Publication date:

2012-03

Permanent link:

https://doi.org/10.3929/ethz-b-000047102

Rights / license:

In Copyright - Non-Commercial Use Permitted

Originally published in:

Biomechanics and Modeling in Mechanobiology 11(3-4), https://doi.org/10.1007/s10237-011-0312-4 


\title{
Mechanisms of reduced implant stability in osteoporotic bone
}

\author{
D. Ruffoni - R. Müller • G. H. van Lenthe
}

Received: 30 March 2010 / Accepted: 26 April 2011 / Published online: 12 May 2011

(c) Springer-Verlag 2011

\begin{abstract}
The determining factors for the fixation of uncemented screws in bone are the bone-implant interface and the peri-implant bone. The goal of this work was to explore the role of the peri-implant bone architecture on the mechanics of the bone-implant system. In particular, the specific aims of the study were to investigate: (i) the impact of the different architectural parameters, (ii) the effects of disorder, and (iii) the deformations in the peri-implant region. A threedimensional beam lattice model to describe trabecular bone was developed. Various microstructural features of the lattice were varied in a systematic way. Implant pull-out tests were simulated, and the stiffness and strength of the bone-implant system were computed. The results indicated that the strongest decrease in pull-out strength was obtained by trabecular thinning, whereas pull-out stiffness was mostly affected by trabecular removal. These findings could be explained by investigating the peri-implant deformation field. For small implant displacements, a large amount of trabeculae in the peri-implant region were involved in the load transfer from implant to bone. Therefore, trabecular removal in this region had a strong negative effect on pull-out stiffness. Conversely, at higher displacements, deformations mainly localized in the trabeculae in contact with the implant; hence, thinning those trabeculae produced the strongest decrease in the strength of the system. Although idealized, the current approach is helpful for a mechanical understanding of the role played by peri-implant bone.
\end{abstract}

D. Ruffoni $(\varangle) \cdot$ R. Müller · G. H. van Lenthe

Institute for Biomechanics, ETH Zurich, Zurich, Switzerland

e-mail: druffoni@ethz.ch

G. H. van Lenthe

Division of Biomechanics and Engineering Design,

K.U.Leuven, Leuven, Belgium
Keywords Implant anchorage - Peri-implant bone architecture - Osteoporosis · Finite element method . Pull-out test

\section{Introduction}

Orthopedic screws are often used in fracture fixation to increase the mechanical stability without the aid of bone cement. Mechanical stability soon after implantation, referred to as primary or initial stability, mainly depends on the quality of the peri-implant bone bed and on the geometrical properties of the implant (Helgason et al. 2008; Zhang et al. 2004, 2006; Chapman et al. 1996). Initial stability has a strong influence on the biological response of osseointegration that follows implant insertion (Puleo and Nanci 1999). It is commonly accepted that high shear stresses and micromotions during the healing process at the bone-implant interface could lead to the formation of a mechanically weak fibrous tissue around the implant (Szmukler-Moncler et al. 1998; Søballe et al. 1992). Key factors for the secondary stability - which is attained at the end of the healing process-are, in addition to the peri-implant region, the amount of bone grown in contact with the implant and the location and the properties of the contact interface (Stadlinger et al. 2007, 2008). Therefore, from a biomechanical viewpoint, the performance of an implant is mainly influenced by the periimplant bone, the bone-implant interface, and the geometry of the implant.

Numerous experimental studies have focused on the role of the implant and of the contact interface: Various surface modifications (Simank et al. 2006; Bernhardt et al. 2005; Wermelin et al. 2008; Peter et al. 2006) as well as new implant designs (Huang et al. 2008; Goldhahn et al. 2006) have been proposed to enhance the contact properties between bone 
and implant. Conversely, less is known about the role of the trabecular bone surrounding the implant. The architecture of the peri-implant bone is obviously of importance for implant anchorage, but its precise role in implant stability is unknown. Gabet et al. (Gabet et al. 2010), in a recent experimental work that combined pull-out tests with micro-computed tomography $(\mu \mathrm{CT})$, showed that secondary implant stability was critically dependent on peri-implant bone; in particular, the microarchitectural parameters that showed the highest correlations with the experimentally measured pull-out stiffness and ultimate force were trabecular thickness $(T b . T h)$ and bone volume fraction $(B V / T V)$. In that study, where titanium screws were implanted in rat tibiae, implant failure was shown to originate in the trabecular bone surrounding the implant at a distance $0.5-1.0 \mathrm{~mm}$ from the implant surface rather than at the bone-implant interface (Gabet et al. 2010). Knowledge on the relative role of peri-implant architecture is therefore crucial to better understand implant failure, especially in osteoporotic bone, where the reduction in mass and the deterioration of architecture could lead to a higher probability of implant loosening (Erdogan et al. 2007; Mori et al. 1997).

Computer simulations, although idealized, offer the possibility of investigating the role of peri-implant architecture. In most of the published computational studies on the mechanical performance of the implant-bone system, the peri-implant bone away from the bone-implant interface has been modeled as a continuum material with homogenized material properties, and only a few studies have explicitly considered the trabecular network (Wirth et al. 2010a; Matsunaga et al. 2010; Tsubota et al. 2003). With the continuum approach, the different bone compartments (i.e. compact bone and trabecular bone) have typically been described using different material constants. The main advantage of this modeling approach consists in the possibility of introducing, within manageable computational time, different levels of material complexity like anisotropy, non-linearity, damage, and time-dependent behavior (Helgason et al. 2008; Zhang et al. 2006; Huang et al. 2008; Natali et al. 2008; Mellal et al. 2004; Natali et al. 2006; Ramamurti et al. 1997). In addition to the peri-implant bone, the bone-implant interface has also been modeled locally by introducing contact elements, frictional effects, cohesive forces, and the possibility of detachment (Wirth et al. 2010b; Huang et al. 2008; Natali et al. 2008; Ko et al. 1996). However, since the internal microarchitecture is not explicitly taken into account, deformations and failure modes typical of cellular materials like trabecular bone are not considered. Moreover, it is known that deformations in trabecular bone are dominated by bending (Nazarian and Müller 2004; Gibson 2005); furthermore, trabecular failure is occurring in well-localized failure bands that probably initiate with buckling of a few trabeculae (Nazarian et al. 2006). Similar failure patterns have recently also been observed during implant pull-out tests (Gabet et al. 2010; Wirth et al. 2010a). Therefore, the main goal of the present work was to explore the role of peri-implant bone architecture on the mechanical properties of the bone-implant system. For this purpose, a three-dimensional beam lattice model to describe the peri-implant trabecular bone was developed. Various microstructural features of the lattice, corresponding to $T b . T h$, trabecular separation $(T b . S p)$, and trabecular number $(T b . N)$, as well as the disorder of the lattice, were varied in a systematic and controlled way. Implant pull-out tests were simulated, and the apparent stiffness and strength of the bone-implant system were computed. In particular, the specific aims of the study were to investigate: (i) the impact of the different architectural parameters on the stiffness and strength of the bone-implant system, (ii) the effects of lattice disorder on calculated stiffness and strength properties, and (iii) to quantify the deformations in the peri-implant region in order to explain the contribution of peri-implant bone to pull-out stiffness and strength.

\section{Materials and methods}

\subsection{Model details}

The foam-like architecture of trabecular bone was idealized as a three-dimensional cubic lattice where each strut represented a single trabecula. The lattice was made up of an array of $17 \times 17 \times 17$ cubic cells (Fig.1a). The implant had a rectangular cuboid shape with a cross section of $3 \times 3 \mathrm{~mm}^{2}$ and was inserted in bone for a reference length of $7 \mathrm{~mm}$ (Fig. 1b). The implant was centered relative to the $x y$-plane of the cubic lattice. An ideally bonded interface was assumed between the implant and the lattice; implant material was considered infinitely rigid. Trabecular bone tissue was modeled with an elasto-plastic material law (Helgason et al. 2008; Verhulp et al. 2008). Young's modulus and yield stress were $10 \mathrm{GPa}$ and $100 \mathrm{MPa}$, respectively. Poisson ratio was 0.3 . In addition, linear strain hardening (slope of $25 \mathrm{MPa}$ ) was included, as suggested by Andrews et al. (Andrews and Gibson 2001), to help simulation convergence. The mechanical pull-out problem was simulated using the commercial finite element (FE) analysis software ABAQUS/standard (Version 6.8-1, Simulia, RI). Each trabecula in the cubic lattice was modeled with 4 Timoshenko beams (element B32 in ABAQUS) that are three-node quadratic elements that account for axial, bending, and shear deformation. This refinement was adequate because the pull-out simulations showed that variation in stiffness and maximum force deviated less than $0.1 \%$ when using more than 4 beam elements per trabecula (data not shown). Circular cross section was used with a reference Tb.Th of $0.3 \mathrm{~mm}$. Reference trabecular separation was $1 \mathrm{~mm}$. The vertical trabeculae that connected the tip of the implant 
Fig. 1 a Three-dimensional cubic lattice model (edge length $17 \mathrm{~mm}$ ) with the implant (in black) positioned in the center of the top $x y$-plane of the bone lattice. b Schematic cross section ( $x z$-plane) of the cubic lattice. Reference implant dimensions are reported. The vertical trabeculae connecting the tip of the implant with the lattice were removed

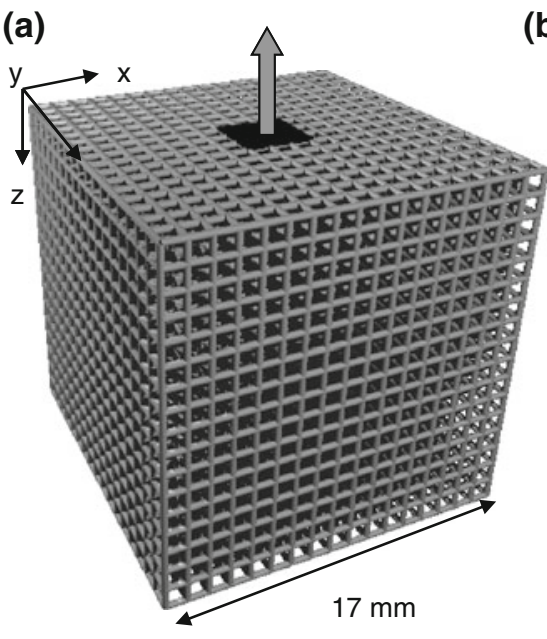

(b)

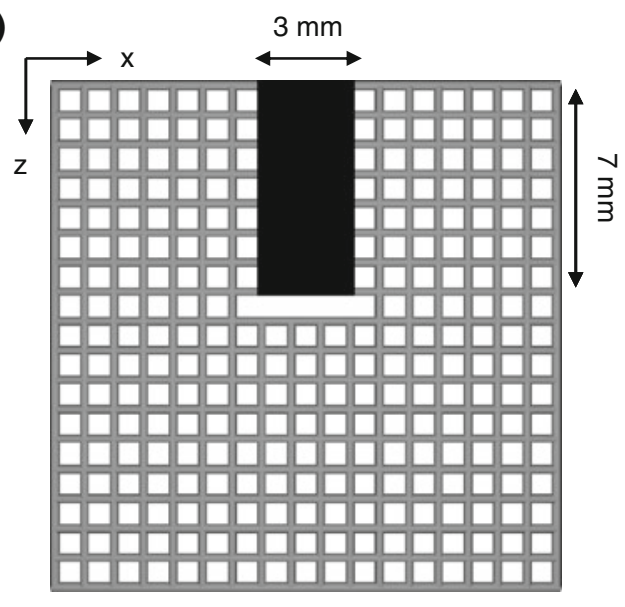

Table 1 Influence of material properties on the pull-out stiffness and strength

\begin{tabular}{lrrr}
\hline Parameter variations & & \multicolumn{2}{c}{ Model predictions (\%) } \\
\cline { 3 - 4 } & & Stiffness & Strength \\
\hline Young's modulus & $-20 \%$ & -20 & -20 \\
\multirow{2}{*}{ Yield stress } & $20 \%$ & 20 & 20 \\
\multirow{2}{*}{ Strain hardening } & $-20 \%$ & 0 & -20 \\
& $20 \%$ & 0 & 20 \\
& $-20 \%$ & 0 & 0 \\
\hline
\end{tabular}

Young's modulus, yield stress and strain hardening were varied $\pm 20 \%$ with respect to the reference values of $10 \mathrm{GPa}, 100 \mathrm{MPa}$ and $25 \mathrm{MPa}$, respectively. The corresponding effects on stiffness and strength were quantified

with the lattice were removed (Fig. 1b). A typical mesh had about 66000 elements and 315000 nodes, and it required about 4 hours to be solved on a Superdome System (Hewlett-Packard, Palo Alto, CA). Implant pull-out experiments were simulated by a kinematic coupling of the displacements of the nodes at the bone-implant interface to a reference node. The reference node was displaced along the $z$-direction, constrained in the $x$ - and $y$-direction and free to rotate, in order to reproduce the boundary conditions normally adopted in experimental tests (Gabet et al. 2006, 2010). The imposed displacement was $10 \%$ of the total size of the cubic bone lattice. The nodes belonging to the bottom surface of the cubic lattice were fixed along $x-, y-$, and $z-$ direction.

\subsection{Parameters investigated}

Considering the uncertainties associated with the material parameters, a sensitivity analysis was performed on the material constants (Young's modulus, yield stress, and strain hardening) that were varied $\pm 20 \%$ (Table 1).
The influence of the insertion depth of the implant was investigated without variations in the peri-implant architecture. Implant length was varied in steps of $2 \mathrm{~mm}$ from a minimum length of $1 \mathrm{~mm}$ to a maximum length of $15 \mathrm{~mm}$ keeping constant the dimensions of the implant cross section and of the overall cubic lattice. $B V / T V$ of the peri-implant trabecular region was estimated by considering the total volume of each individual trabecula divided by the apparent volume of the whole sample (Guo and Kim 2002; Yeh and Keaveny 1999):

$$
\frac{B V}{T V}=\frac{\frac{\pi}{4} \sum_{i=1}^{T b . N} T b . T h_{i}^{2} L_{i}}{V_{\text {sample }}}
$$

where $T b . N$ is the total number of trabeculae contained in the sample before placing the implant, and $L$ is the trabecular length. To simulate the reduction in relative density, three different mechanisms were considered, which decreased $B V / T V$ approximately by the same amount (Table 2): first, $T b . T h$ was uniformly reduced; second, $T b . S p$ was increased; and third, Tb.N was decreased by a random removal of trabeculae. Trabecular elements were removed from the bulk of the lattice and without creating unconnected parts. For each reduction in relative density due to trabecular loss, 5 different structures were generated. In all three scenarios, $B V / T V$ was reduced by $5 \%, 15 \%, 25 \%$, and $35 \%$ starting from an initial value for the intact lattice of $23.8 \%$ Structural disorder was introduced in the cubic lattice by shifting the trabecular intersections in random directions by a fixed amount $\alpha$ (Yeh and Keaveny 1999; Luxner et al. 2009; Jensen et al. 1990). Only intersections with a coordination number 6 (i.e., with 6 trabeculae coming together at the intersection) were perturbed. Different perturbation magnitudes were considered, which corresponded to $10 \%, 20 \%, 30 \%$, and $40 \%$ of the reference Tb.Sp of $1 \mathrm{~mm}$. For each value of the perturbation parameter $\alpha, 5$ different structural realizations were generated and solved with FE. 
Table 2 Reduction in bone relative density by three different bone loss mechanisms

\begin{tabular}{llll}
\hline$B V / T V$ loss & $\begin{array}{l}\text { Mechanism (A) } \\
\text { Tb.Th }[\mathrm{mm}]\end{array}$ & $\begin{array}{l}\text { Mechanism (B) } \\
\text { Tb.Sp }[\mathrm{mm}]\end{array}$ & $\begin{array}{l}\text { Mechanism (C) } \\
\text { Tb.N [-] }\end{array}$ \\
\hline$-5 \%$ & $0.292(-2.7 \%)$ & $1.026(2.6 \%)$ & $15698(-4.9 \%)$ \\
$-15 \%$ & $0.277(-7.7 \%)$ & $1.085(8.5 \%)$ & $14045(-15 \%)$ \\
$-25 \%$ & $0.260(-13 \%)$ & $1.155(15 \%)$ & $12393(-25 \%)$ \\
$-35 \%$ & $0.242(-19 \%)$ & $1.240(24 \%)$ & $10741(-35 \%)$ \\
$-(22 \div 38) \%^{\mathrm{a}, \mathrm{b}}$ & $-(7 \div 15) \%^{\mathrm{b}}$ & $(17 \div 30) \%^{\mathrm{a}, \mathrm{b}}$ & $-(11 \div 22) \%^{\mathrm{a}, \mathrm{b}}$
\end{tabular}

(A) reduction in trabecular thickness $(T b . T h)$; (B) increase in trabecular separation $(T b . S p)$; and $(\mathrm{C})$ reduction in trabecular number $(T b . N)$ by removal of entire trabeculae. Indicated are the architectural parameters that result in identical reductions in bone volume fraction $(B V / T V)$ and the relative variation in those parameters. Note that in the reference configuration, $T b . T h$ was $0.3 \mathrm{~mm}$ and $T b . S p$ was $1 \mathrm{~mm}$. In the last row are reported the percentage variations in architectural parameters measured at ${ }^{\mathrm{a}}$ lumbar spine (Stauber and Müller 2006) and ${ }^{b}$ femoral neck (Chen et al. 2010)

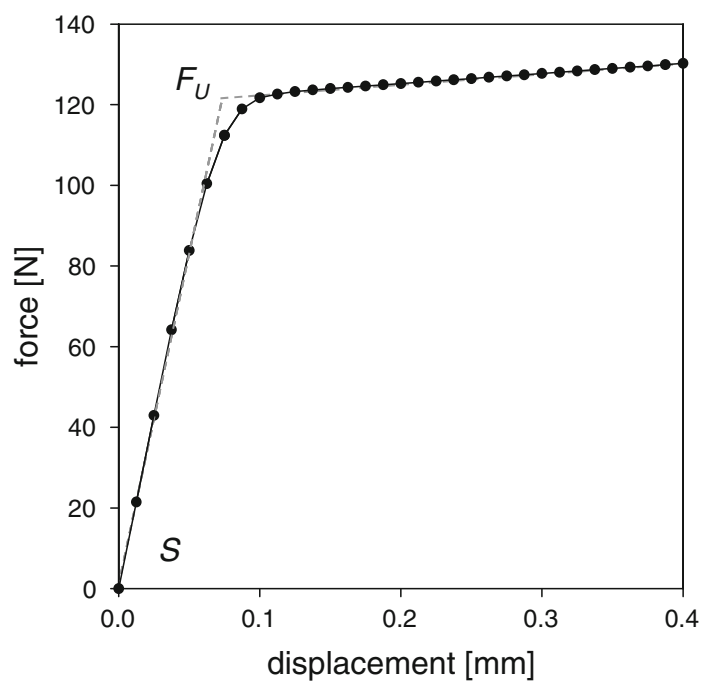

Fig. 2 Characteristic force-displacement curve of a simulated implant pull-out test. Stiffness $(S)$ was defined as the slope of the linear part of the force displacement curve; the ultimate force $\left(F_{U}\right)$ was defined as the intersection point between the region of linear elasticity and the region of constant strain hardening (Andrews and Gibson 2001)

\subsection{Model outcome}

The two main mechanical parameters derived from the pullout simulations were stiffness, $S$, and ultimate force, $F_{U}$, of the bone-implant system. The stiffness was measured as the slope of the linear part of the force-displacement curve, whereas the ultimate force (or strength) was described as the intersection point between the region of linear elasticity and the region of constant strain hardening (Andrews and Gibson 2001) (Fig. 2). This definition has the advantages of being independent from the specific shape of the forcedisplacement curve in the yield region and of being unaffected by numerical issues related to the number of steps taken by the FE solver. The peri-implant deformation field was investigated both at small implant displacements $(d<$ $0.06 \mathrm{~mm}$; linear elastic region) and at large implant displacements ( $d>0.125 \mathrm{~mm}$; plastic deformations). For this purpose, an averaged displacement, $U_{l, z}$, was computed for each concentric layers around the implant $(l=0, \ldots, 7)$ and at different depths $(z=0, \ldots, 17)$ (Fig. 6a, c). Considering the current model geometry, in each horizontal $x y$-plane, 8 different layers were present: layer number 0 was the innermost layer in contact with the implant, whereas layer number 7 was the outermost layer. $U_{1,0}$ gave, for example, the amount of displacement averaged in the first concentric layer and in the top plane of the lattice.

\section{Results}

3.1 Force-displacement behavior, material properties, and implant insertion depth

A representative force-displacement curve of a pull-out simulation, obtained considering the unperturbed lattice with an implant insertion depth of $7 \mathrm{~mm}$, presented three features (Fig. 2). A linear elastic region, up to an implant displacement of about $0.06 \mathrm{~mm}$, was followed by a transition region where the force increased non-linearly with the displacement. For a displacement larger than about $0.125 \mathrm{~mm}$, a region of constant slope, indicating fully developed strain hardening, was attained. Qualitatively similar plots resulted from disordered lattices or lattices with reduced relative density. The variations in the pull-out stiffness and ultimate force for different material constants are reported in Table 1. Variations in the tissue modulus were linearly correlated with the model stiffness and strength for the considered range. Yield stress only affected pull-out strength: Changes in strength were in the same direction and of the same magnitude as the variations in yield stress. The slope of the strain hardening region had no influence on stiffness and strength.

Implant insertion depth influenced both stiffness and ultimate force in approximately the same linear fashion, with a slope of $0.125 \mathrm{~mm}^{-1}$ for stiffness and of $0.105 \mathrm{~mm}^{-1}$ for ultimate force (Fig. 3).

\subsection{Influence of relative density reduction}

Simulated reductions in $B V / T V$ led to decreases in calculated pull-out stiffness and strength. The reduction in both stiffness and ultimate force was dependent on the way the bone loss occurred (Fig. 4). The strongest decrease in the pull-out stiffness was observed when bone loss was due to a random removal of trabeculae (Fig. 4a): A 35\% reduction in relative density caused a decrease in stiffness of about $71 \%$. 


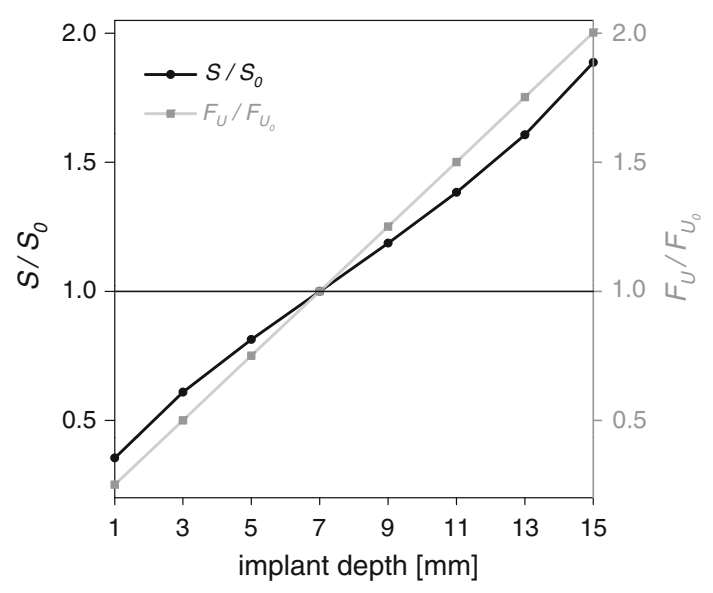

Fig. 3 Influence of implant insertion depth on the stiffness $(S)$ and ultimate force $\left(F_{U}\right)$ of the bone-implant system. Data were normalized by the respective values $\left(S_{0}, F_{U_{0}}\right)$ at the reference implant depth of $7 \mathrm{~mm}$

The same amount of bone loss reduced stiffness by $50 \%$ and $38 \%$ in case of uniform trabecular thinning and increase in trabecular spacing, respectively. Trabecular thinning was the mechanism that most affected the ultimate pull-out force, followed by trabecular removal and trabecular spacing (Fig. 4b). A density reduction of $35 \%$ led to decreases in ultimate force of about $50 \%$ when decreasing $T b . T h, 35 \%$ when decreasing $T b . N$ and $24 \%$ when increasing $T b . S p$.

\subsection{Influence of disorder}

The amount of disorder present in the peri-implant region influenced pull-out stiffness and strength in the same fashion but with quite different magnitudes (Fig. 5). Structural perturbation had a limited effect on stiffness: Increasing the perturbation to a maximum value of $40 \%$ of the reference trabecular spacing $(1 \mathrm{~mm})$ only caused an increase of about $13 \%$ in stiffness (Fig. 5). The effect on ultimate force was much stronger: The same perturbation magnitude of $40 \%$ increased the ultimate force by $80 \%$ (Fig. 5).

\subsection{Deformation field}

The magnitude and the distribution of the deformation inside the peri-implant bone region were evaluated for a lattice without any structural perturbations. In the linear elastic regime $(d<0.06 \mathrm{~mm})$, the averaged displacement in the first concentric layer on the top plane, $U_{1,0}$, was approximately $40 \%$ less than the displacement of the implant $U_{0,0}$ (Fig. 6b). At the second layer, $U_{2,0}$ was only approximately $25 \%$ of the implant displacement; from the fourth layer on, the magnitude of the averaged layer displacement was less
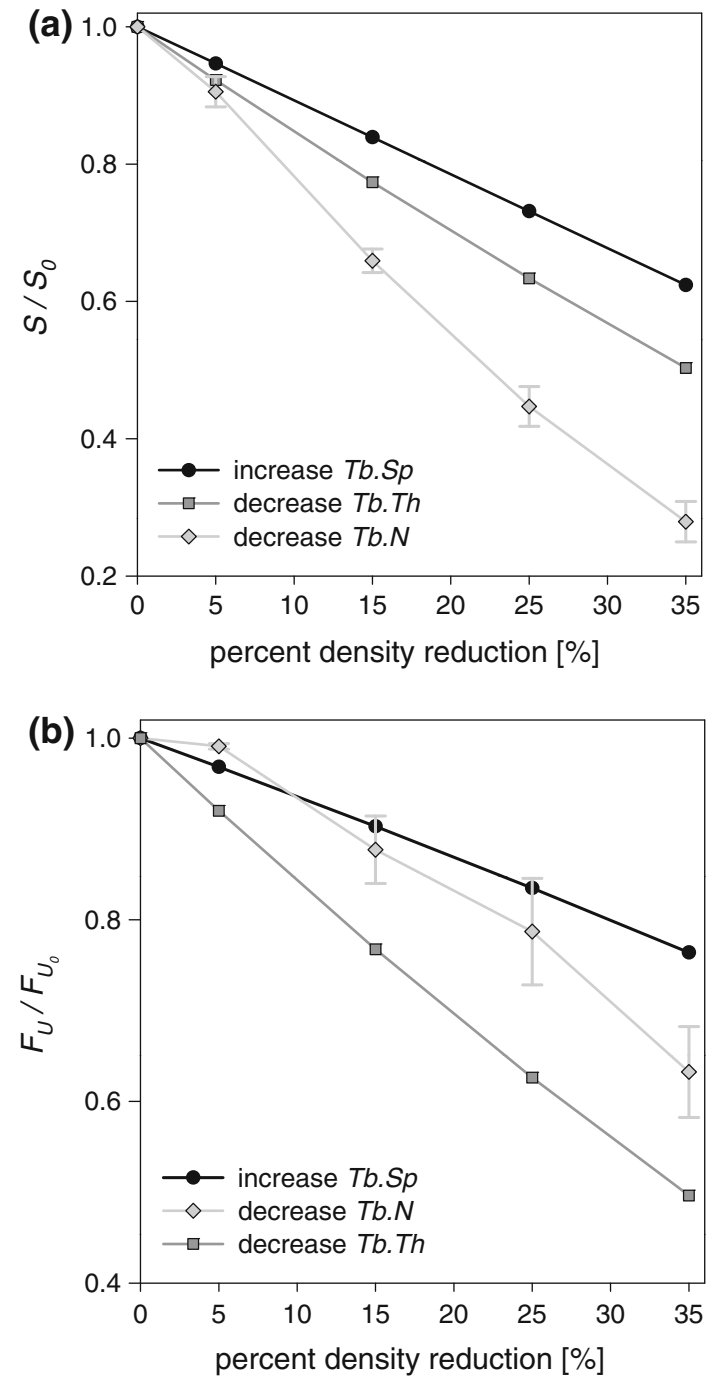

Fig. 4 Effects of relative density reduction by three different mechanisms on a stiffness and $\mathbf{b}$ ultimate force normalized by the respective values of the reference situation. For the case of trabecular removal (decreased $T b . N$ ), data points represent the average values over 5 different realizations and error bars denote one standard deviation interval

than $6 \%$ of the imposed pull-out displacement. For larger implant displacements $(d>0.125 \mathrm{~mm})$, where plastic deformations started to occur, the averaged displacement in the peri-implant region decayed extremely fast when moving away from the implant surface (Fig. 6b): The averaged displacement of the first layer, $U_{1,0}$, was only $3.5 \%$ of the displacement at the implant surface. At the second layer, $U_{2,0}$ was less than $1 \%$ of the implant displacement.

A different trend was observed when considering the same layer (e.g., layer 0) at different lattice depths $z$ (Fig. 6d). For values of the $z$-coordinate less or equal to the implant insertion depth $(z \leq 7 \mathrm{~mm})$, the averaged displacements were approximately constant. Conversely, in the region below the tip of the implant $(8 \mathrm{~mm} \leq z \leq 17 \mathrm{~mm})$, the averaged 


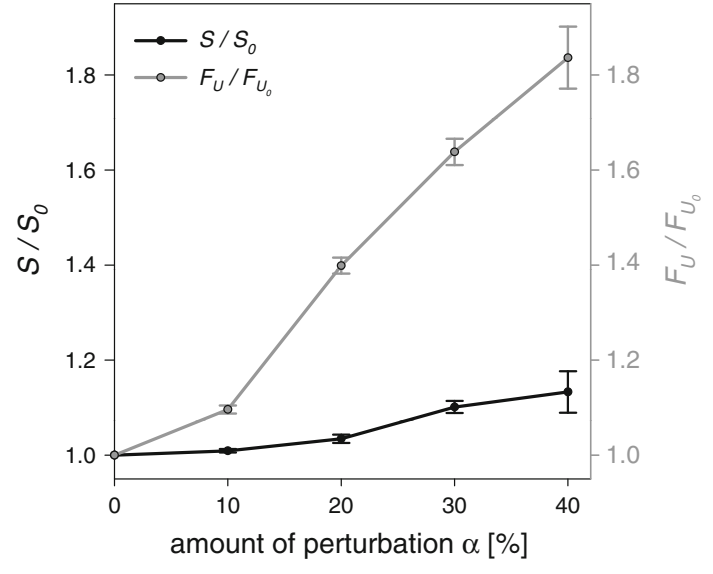

Fig. 5 Effects of structural perturbations on stiffness and ultimate force. Data points represent the average values over 5 different realizations, and error bars denote one standard deviation interval. Results were normalized by the respective values of the unperturbed case

layer displacements were basically 0 for $d>0.125 \mathrm{~mm}$; for $d<0.06 \mathrm{~mm}$ the displacement below the tip of the implant was approximately $20 \%$ of the implant displacement and then decayed linearly to zero at the boundary of the model (Fig. 6d).

\section{Discussion}

In this study, we developed a computational cellular solids model to investigate the role of peri-implant trabecular architecture on the mechanics of the bone-implant system. The main focus of the study was to quantify how different architectural parameters affect pull-out stiffness and strength with the aim of identifying the most critical architectural aspect that, especially in osteoporotic bone, may compromise implant stability. Although idealized, the use of cellular solids is a proven modeling approach for understanding the basic mechanisms that govern the mechanics of trabecular bone (Gibson 2005; Gibson and Ashby 1997). Recently, it was validated against experimental tests and micro-finite element analysis as a fast tool to predict trabecular bone mechanical properties (van Lenthe et al. 2006). The method has been successfully applied in several studies: for example, to examine the impact of bone loss and bone recovery on trabecular stiffness and strength (Guo and Kim 2002; Vajjhala et al. 2000) or to determine the influence of variations in $T b . T h$ (Yeh and Keaveny 1999). The concepts of cellular solids were applied here for the first time to analyze bone-implant constructs. In our study, we have tried not to
Fig. 6 a Schematic view of the top plane of the lattice with the 7 concentric layers used to compute the averaged displacements; the implant, in gray, is also visible. b Variations in the averaged displacement normalized by the displacement of the implant $\left(U_{l, 0} / U_{0,0}\right)$ for the different layer on the top plane. c Schematic view of the middle cross section of the lattice in the $x z$-plane; layer 0 at different depths along the $z$-coordinate is highlighted. Again, the implant is visible in gray. $\mathbf{d}$ The averaged displacement for layer 0 normalized by the displacement of the implant $\left(U_{0, z} / U_{0,0}\right)$ is shown for different depth ( $z$-coordinate). Curves are shown both for small $(d<0.06 \mathrm{~mm})$ and large $(d>0.125 \mathrm{~mm})$ applied implant displacements

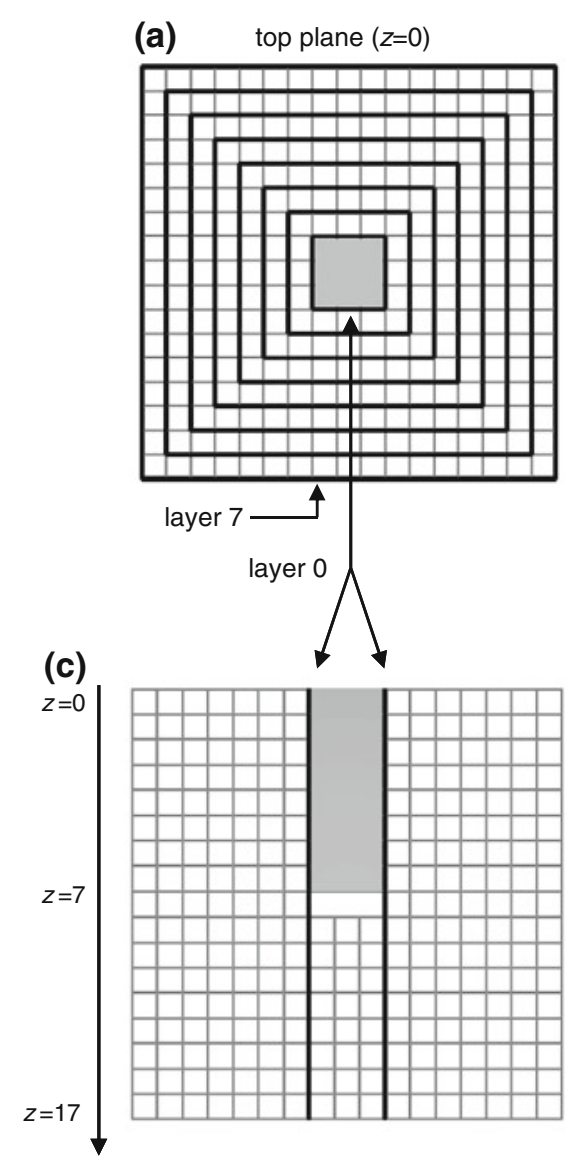

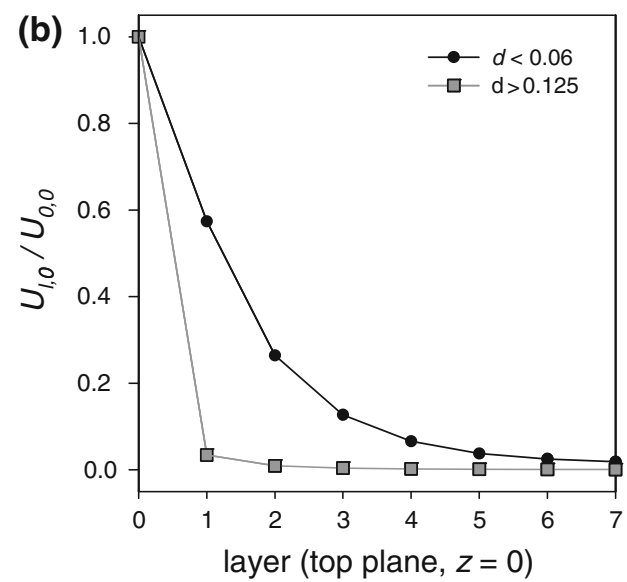

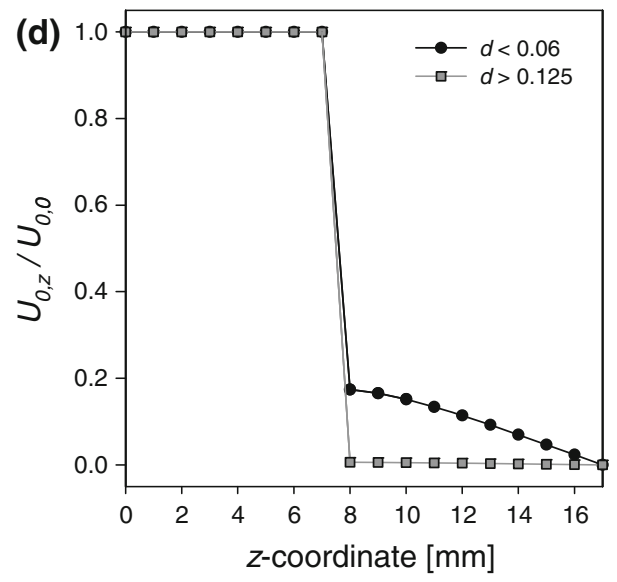


alter the main modeling aspects of cellular solids; in fact, the implant and the bone-implant interface were idealized in order to focus on the role of microarchitecture. In addition, it has to be realized that the mechanical properties of the boneimplant interface are still not well known and their mechanical origin is not well understood. Therefore, we decided not to include those uncertainties.

In our model, the trabeculae were perfectly bonded to the implant surface. Assuming infinite shear modulus and strength at the interface can lead to an overestimation of stiffness and strength of the bone-implant construct as well as of the importance of peri-implant bone. However, the magnitude of such overestimation and the specific role of the interface might be quite different depending on the type of bone compartment investigated. In cortical bone, it is well accepted that the interface plays a major role: The amount of bone in contact with the implant as well as the frictional properties at the contact interface are important parameters that influence the mechanics of the bone-implant system (Huang et al. 2008). Implant stability in trabecular bone is less well understood. Considering the reduced contact surface between trabecular bone and implant, it has been suggested that macroscopic phenomena like mechanical interlocking between single trabeculae and implant may dominate over microscopic events like friction (Wirth et al. 2010a,b). Furthermore, in case of well-osseointegrated implants, there is experimental evidence that failure does not occur at the interface but in the trabecular network (Gabet et al. 2010). For these cases, our assumption of a fully bonded interface will be a good modeling approach. In addition, we recently demonstrated that even when no osseointegration is present, the assumption of a fully bonded interface is appropriate. Specifically, in an ex-vivo study, screws were implanted in trabecular bone, and pull-out strength was measured. Pull-out strength was also quantified using specimen-specific micro-structural finite element analysis. We showed that even when assuming a fully bonded interface, pull-out strength was predicted very well $\left(R^{2}=0.87\right)$ (Wirth et al. 2010a). To the best of our knowledge, computational models including both a direct modeling of the discrete trabecular network as well as a detailed numerical description of the interface have not been presented so far. Modeling strategies that have been proposed for the boneimplant interface range from a perfect bonding to the inclusions of friction, cohesive forces, and possible detachment if stresses at the interface overcome a predetermined threshold (Wirth et al. 2010a; Natali et al. 2008). Increasing the complexity of the numerical description of the interface would imply that more complex parameters (such as the shear stiffness and the ultimate shear strength of the interface) need to be provided as input. However, the bonding characteristics depend on many factors, and values for the bonding strength in the range from 1 to $20 \mathrm{MPa}$ have been reported (Natali et al.
2008). Another important issue that probably influences the mechanics at the interface is the amount of stresses resulting from the implantation procedure (Søballe et al. 1991, 1992); these are difficult to be quantified let alone measured. Moreover, implant surface roughness could play an important role for osseointegration and anchorage, particularly in connection with frictional forces and pre-stresses.

The implant was attached to the lattice only through horizontal trabeculae; The vertical trabeculae connecting the tip of the implant with the lattice were removed (Fig. 1b) to avoid the generation of high tensile stresses. In a primary implant stability scenario, these trabeculae will not play a role in supporting the screw during a pull-out test. In a scenario testing the secondary implant stability, these trabeculae may contribute to load transfer; but, in general, the tip of the implant screw is tapered and quite small, hence the contribution of these trabeculae would only be small. The implant surface was assumed smooth and screw threads were not considered. In fact, with the idealized fully bonded interface that we assumed for our model, such features will not contribute to the mechanics of the system. The load applied to the implant is redistributed inside the trabecular network depending on bone architecture, implant geometry, and material properties. Differences in stiffness between implant material and bone tissue may result in a quite different mechanical behavior of the bone-implant system. For instance, if the elastic properties of the implant and the bone are comparable, the applied load will be carried only by a limited portion of bone (Zhang et al. 2004; Grewal and Sabbaghian 1997). Our modeling strategy corresponded to an infinitely rigid implant material. This ensured uniform load redistribution along the entire implant length; all the trabeculae in contact with the implant were subject to the same displacement, which equaled the displacement imposed to the implant. This displacement behavior is in agreement with more realistic scenarios where the Young's modulus of the implant is usually more than one order of magnitude bigger that the Young's modulus of bone (Wirth et al. 2010a; Zhang et al. 2004).

The force-displacement behavior obtained with our computer simulations (Fig. 2) differs in part from the one resulting from pull-out experiments. After a linear increase in force, the experimental curves often display a peak that is followed by a drop in force (An and Draughn 2000). This pattern may be caused by highly non-linear failure mechanisms at the trabecular level (e.g., buckling or crack formation) and by slip at the bone-implant interface. Furthermore, the fragile mechanical behavior of trabecular bone under tensile stress is not accounted for in the elasto-plastic material model used. One of the main difficulties related with modeling trabecular bone failure as well as failure at the bone-implant interface is that those mechanisms are only partially understood and that suitable numerical models for bone behavior in the post-yield region are still very much debated. It is specifically for this 
reason that we did not investigate the post-yield behavior of the bone-implant constructs, but only considered the mechanical behavior up to the yield point. Our simulations, after yield, did not capture the experimental failure pattern but showed a region of strain hardening with constant slope. Nevertheless, at the apparent level, the model did capture the stiffness and strength aspects that are the two most important parameters used to quantify implant stability. Additionally, we performed a sensitivity analysis to assess the role of the material constants on the model outcomes. Pull-out stiffness and strength, when influenced, scaled linearly with the variations in the material parameters, thus confirming the robustness of our results with respect to uncertainties of the material behavior. The reference material parameters were varied by $20 \%$, which only cover a small range of the tissue level properties of trabecular bone reported in the literature. However, such range is large enough to grasp the qualitative and quantitative role of material properties on the mechanical behavior of our model, so no further variations in the material properties were introduced. Based on experimental evidence that at the end of the osseointegration process (i.e., secondary stability scenario), the mechanical properties of new bone formed in contact with the implant do not differ from the properties of mature bone (Ballarre et al. 2010), we did not consider differences in the tissue properties as a function of the distance from the implant surface. Moreover, in real trabecular bone, significant variations of the cross section along the trabecular axis might be present. Therefore, a real trabecular network will have a different failure behavior than a network where all the trabeculae have a uniform thickness. At the local level, however, it is still not understood whether failure will initiate at the weakest trabecula with the smallest cross section, as would be the case when considering each trabecula individually and isolated from the trabecular network (Ruffoni et al. 2010).

Our model showed that pull-out strength increased linearly with implant insertion depth (Fig. 3). Such results were also obtained in other studies by Zhang et al. (Zhang et al. 2004, 2006), which, however, used a different modeling approach for trabecular bone and considered a different range of implant lengths. In our work, the trabeculae were explicitly modeled, and the increase in implant length was in the range of $1-15 \mathrm{~mm}$ in steps of $2 \mathrm{~mm}$ increases. Conversely, Zhang and co-workers described the peri-implant trabecular bone with an equivalent continuum material, and they considered a two-fold increase in screw length (from $16.5 \mathrm{~mm}$ to $38.5 \mathrm{~mm}$ ). Implant stiffness also increased almost linearly with implant length with a somewhat less regular behavior than strength.

The effects of three density reduction mechanisms were examined, and the variations in the model parameters (Table 2) were chosen to represent realistic microarchitectural modifications occurring with aging and osteoporosis.
Obviously, we could not depict all the variations in structural indices at each skeletal location; therefore, we focused on modeling changes occurring at the femoral head and at the lumbar spine which, together with the distal radius, are sites where most of the osteoporotic fracture occurs (Cummings et al. 1989). For those two locations, our model parameters cover quite well the range of variations in the trabecular indices reported in literature (Table 2). It is well accepted that bone microarchitecture changes differently at different skeletal locations. At lumbar spine, for instance, a bone loss of $38 \%$ over a time period of 60 years has been reported (Stauber and Müller 2006). In the same study, $T b . N$ decreased by $22 \%$, whereas $T b . S p$ increased by $30 \%$. In a different work, which considered the femoral neck and a slightly shorter time frame (40 years), $B V / T V$ decreased by $22 \%, T b . T h$ by $15 \%$, and $T b . N$ by $11 \%$; conversely, $T b . S p$ increased by $17 \%$ (Chen et al. 2010). In our study, we have simulated a maximum bone loss of $35 \%$, which corresponded to either a decrease in $T b . T h$ by $20 \%$, a decrease in $T b . N$ by $35 \%$, or an increase in $T b . S p$ by $24 \%$. The strongest decrease in pull-out stiffness was caused by randomly removing trabeculae from the peri-implant bone region. Pull-out strength was most affected by trabecular thinning. Although the analysis was performed on the regular lattice, the same trend was present when applying the three different density reduction mechanisms also on the lattices with structural disorder (amount of perturbation 20\%, data not shown). Furthermore, considering the behavior of pull-out stiffness and strength as a function of relative density (Fig. $4 a$ and b), it is expected that a further decrease in relative density will not change the qualitative trend of our results. Hence, simulating more bone loss with bigger variations in the geometrical parameters will not produce more insights into the behavior of our model. The most severe scenario we analyzed, which was a reduction in relative density of $35 \%$, caused a decrease in stiffness and in ultimate force of about $71 \%$ and $50 \%$, respectively. This behavior is only in partial agreement with previous findings on 2D and 3D cellular solid models for trabecular bone, which always predicted trabecular loss as the worst scenario both for stiffness and strength (Guo and Kim 2002; Vajjhala et al. 2000; Silva and Gibson 1997). It should be noted, however, that we modeled bone-implant behavior, whereas previous models investigated trabecular bone properties only. The discrepancy between previous studies and our findings on the strength behavior can be explained by the different loading conditions and the specific bone deformations around the implant (Fig. 6b). In the case of implant pull-out simulations for small displacements, a large amount of trabeculae in the peri-implant region were involved in the load transfer from the implant to the bone lattice. Therefore, random trabecular removal, which mainly occurred in the peri-implant region, had a strong negative effect on implant pull-out stiffness. Conversely, at higher displacements, deformations mainly 
localized in the first layer of trabeculae in contact with the implant (Fig. 6b), hence thinning those trabeculae produced the strongest decrease in the strength of the system.

In order to account for the variability in the trabecular arrangement, structural disorder was introduced by perturbing the position of the trabecular intersection. We found that a perturbation of $40 \%$ resulted in a stiffness increase of $13 \%$ only. This modest effect of disorder on the pull-out stiffness can be explained by considering the deformation mechanism. During the pull-out simulations of the unperturbed lattice, the pulled trabeculae behaved like cantilever beams: They deformed mainly in bending, and they induced rotations at the neighboring joints. Introducing structural perturbations did not substantially change the bending-dominated deformations. In contrast, when disorder is introduced in regular cubic lattices (without an implant) that are tested in compression, the deformation mechanism changes form beam-compression to beam-bending so that the overall apparent stiffness decreases drastically (Luxner et al. 2009; Jensen et al. 1990). For the ultimate pull-out force, the role of disorder was stronger: Lattices with $40 \%$ perturbations showed an almost $80 \%$ higher pull-out force. In the reference lattice, all the trabeculae coupled with the reference node reached the yield point at the same time. In the disordered structures, load was unevenly redistributed and this prevented the formation of localized regions with yielded trabeculae. This finding is in agreement with previous works on cellular structures (Luxner et al. 2007, 2009). It should be mentioned that a secondary effect of increasing disorder was an increase in the relative density of the lattice due to an increase in the average length of individual trabeculae. Hence, a perturbation magnitude of $40 \%$ resulted in a relative density increase of about $7 \%$. This could partially contribute to the observed stiffness increase but, obviously, cannot be the main factor responsible for the higher pull-out force. Another effect of introducing lattice disorder and random trabecular removal was the breaking of the symmetry of the model and, therefore, an increase in the forces needed to constrain the implant displacement in $x-$ and $y$ - direction. The magnitude of those forces increased with the amount of disorder and with the number of trabeculae removed; however, it was always much smaller than the force along the pull-out direction $(z-$ direction). The maximum force observed in the $x y$-plane for the most disordered lattice (perturbation of $40 \%$ ) was only $4.6 \%$ of the total axial pull-out force, whereas for the random removal scenario corresponding to a bone loss of $35 \%$, the maximum force in the $x y$-plane was $3.7 \%$ of the ultimate pull-out force.

In summary, a beam finite element model was developed to perform a parametric investigation into how bone microarchitectural changes impact implant pull-out properties. The importance of considering individual architectural elements in stiffness and strength assessment of normal and osteoporotic bone has already been demonstrated in various studies (Liu et al. 2009a,b; Stauber et al. 2006). Here, we showed that individual trabeculae have a pivotal role in implant fixation as well. Pull-out stiffness strongly decayed when trabeculae were randomly removed from the peri-implant network, whereas the behavior of pullout strength was dictated by the thickness of the trabeculae in contact with the implant. The main outcomes of our idealized bone-implant model are in agreement with recent experimental findings on the mechanical stability of osseointegrated implants. Specifically, Gabet and colleagues (Gabet et al. 2010) assessed the pull-out strength of osseointegrated implants in trabecular bone of rats. In that study, it has been shown that trabecular thickness is the architectural quantity that best correlated with implant pull-out strength; similar findings, along with a detailed investigation of the displacement field, were reported in our study. Our results show that potential successful treatment to increase implant strength should aim at restoring trabecular thickness, for example by use of a bone anabolic agent such as PTH (Gabet et al. 2010). We hypothesize that the present modeling strategy could also be applied to a more realistic representation of the trabecular network, obtained, for example, by combining $\mu \mathrm{CT}$ images and skeletonization techniques (van Lenthe et al. 2006; Liu et al. 2009a) to give a fast specimen-based prediction of implant stability in osteoporotic bone.

Acknowledgments This research was funded by the AO Foundation (network grant CPP1).

\section{References}

An Y, Draughn R (2000) Mechanical testing of bone and the boneimplant interface. CRC Press, Boca Raton

Andrews E, Gibson L (2001) The influence of cracks, notches and holes on the tensile strength of cellular solids. Acta Mater 49(15): 2975-2979

Ballarre J, Manjubala I, Schreiner WH, Orellano JC, Fratzl P, Ceré S (2010) Improving the osteointegration and boneimplant interface by incorporation of bioactive particles in solgel coatings of stainless steel implants. Acta Biomater 6:1601-1609

Bernhardt R, van den Dolder J, Bierbaum S, Beutner R, Scharnweber D, Jansen J, Beckmann F, Worch H (2005) Osteoconductive modifications of ti-implants in a goat defect model: characterization of bone growth with sr $\mu$ ct and histology. Biomaterials 26(16): 3009-3019

Chapman JR, Harrington R, Lee K, Anderson P, Tencer A, Kowalski D (1996) Factors affecting the pullout strength of cancellous bone screws. J Biomech Eng 118(3):391-398

Chen H, Zhou X, Shoumura S, Emura S, Bunai Y (2010) Age- and gender-dependent changes in three-dimensional microstructure of cortical and trabecular bone at the human femoral neck. Osteoporos Int 21:227-236

Cummings S, Black D, Rubin S (1989) Lifetime risks of hip, colles, or vertebral fracture and coronary heart-disease among white postmenopausal women. Arch Intern Med 149(11):2445-2448 
Erdogan O, Shafer D, Taxel P, Freilich M (2007) A review of the association between osteoporosis and alveolar ridge augmentation. Oral Surg Oral Med Oral Pathol Oral Radiol Endod 104(6):738-746

Gabet Y, Müller R, Levy J, Dimarchi R, Chorev M, Bab I, Kohavi D (2006) Parathyroid hormone 1-34 enhances titanium implant anchorage in low-density trabecular bone: a correlative micro-computed tomographic and biomechanical analysis. Bone 39(2):276-282

Gabet Y, Kohavi D, Voide R, Mueller T, Müller R, Bab I (2010) Endosseous implant anchorage is critically dependent on mechanostructural determinants of peri-implant bone trabeculae. J Bone Miner Res 25(3):575-583

Gibson L (2005) Biomechanics of cellular solids. J Biomech 38(3): 377-399

Gibson L, Ashby M (1997) Cellular solids: structure and properties. Cambridge University Press, Cambridge

Goldhahn J, Neuhoff D, Schaeren S, Steiner B, Linke B, Aebi M, Schneider E (2006) Osseointegration of hollow cylinder based spinal implants in normal and osteoporotic vertebrae: a sheep study. Arch Orthop Trauma Surg 126(8):554-561

Grewal A, Sabbaghian M (1997) Load distribution between threads in threaded connections. J Pressure Vessel Technol 119(1):91-95

Guo X, Kim C (2002) Mechanical consequence of trabecular bone loss and its treatment: a three-dimensional model simulation. Bone 30(2):404-411

Helgason B, Viceconti M, Runarsson TP, Brynjolfsson S (2008) On the mechanical stability of porous coated press fit titanium implants: a finite element study of a pushout test. J Biomech 41(8):16751681

Huang H, Hsu J, Fuh L, Tu M, Ko C, Shen Y (2008) Bone stress and interfacial sliding analysis of implant designs on an immediately loaded maxillary implant: a non-linear finite element study. J Dent 36(6):409-417

Jensen K, Mosekilde L, Mosekilde L (1990) A model of vertebral trabecular bone architecture and its mechanical-properties. Bone 11(6):417-423

Ko CC, Kohn D, Hollister S (1996) Effective anisotropic elastic constants of bimaterial interphases: comparison between experimental and analytical techniques. J Mater Sci 7:109-117

Liu X, Bevill G, Keaveny T, Sajda P, Guo X (2009a) Micromechanical analyses of vertebral trabecular bone based on individual trabeculae segmentation of plates and rods. J Biomech 42(3):249256

Liu X, Zhang X, Guo X (2009b) Contributions of trabecular rods of various orientations in determining the elastic properties of human vertebral trabecular bone. Bone 45(2):158-163

Luxner M, Stampfl J, Pettermann H (2007) Numerical simulations of $3 \mathrm{~d}$ open cell structures-influence of structural irregularities on elasto-plasticity and deformation localization. Int J Solids Struct 44(9):2990-3003

Luxner M, Woesz A, Stampfl J, Fratzl P, Pettermann H (2009) A finite element study on the effects of disorder in cellular structures. Acta Biomater 5(1):381-390

Matsunaga S, Shirakura Y, Ohashi T, Nakahara K, Tamatsu Y, Takano N, Ide Y (2010) Biomechanical role of peri-implant cancellous bone architecture. Int J Prosthodont 23:333-338

Mellal A, Wiskott H, Botsis J, Scherrer S, Belser U (2004) Stimulating effect of implant loading on surrounding bone-comparison of three numerical models and validation by in vivo data. Clin Oral Implant Res 15(2):239-248

Mori H, Manabe M, Kurachi Y, Nagumo M (1997) Osseointegration of dental implants in rabbit bone with low mineral density. J Oral Maxil Surg 55(4):351-361

Natali A, Pavan P, Ruggero A (2006) Analysis of bone-implant interaction phenomena by using a numerical approach. Clin Oral Implant Res 17(1):67-74
Natali A, Carniel E, Pavan P (2008) Investigation of bone inelastic response in interaction phenomena with dental implants. Dent Mater 24(4):561-569

Nazarian A, Müller R (2004) Time-lapsed microstructural imaging of bone failure behavior. J Biomech 37(1):55-65

Nazarian A, Stauber M, Zurakowski D, Snyder B, Müller R (2006) The interaction of microstructure and volume fraction in predicting failure in cancellous bone. Bone 39(6):1196-1202

Peter B, Gauthier O, Laib S, Bujoli B, Guicheux J, Janvier P, van Lenthe G, Müller R, Zambelli P, Bouler J, Pioletti D (2006) Local delivery of bisphosphonate from coated orthopedic implants increases implants mechanical stability in osteoporotic rats. J Biomed Mater Res A 76(1):133-143

Puleo D, Nanci A (1999) Understanding and controlling the boneimplant interface. Biomaterials 20(23-24):2311-2321

Ramamurti B, Orr T, Bragdon C, Lowenstein J, Jasty M, Harris W (1997) Factors influencing stability at the interface between a porous surface and cancellous bone: a finite element analysis of a canine in vivo micromotion experiment. J Biomed Mater Res 36(2):274-280

Ruffoni D, Dunlop J, Fratzl P, Weinkamer R (2010) Effect of minimal defects in periodic cellular solids. Philos Mag 90:1807-1818

Silva M, Gibson L (1997) The effects of non-periodic microstructure and defects on the compressive strength of two-dimensional cellular solids. Int J Mech Sci 39(5):549-563

Simank H, Stuber M, Frahm R, Helbig L, van Lenthe H, Müller R (2006) The influence of surface coatings of dicalcium phosphate (dcpd) and growth and differentiation factor-5 (gdf-5) on the stability of titanium implants in vivo. Biomaterials 27(21):39883994

Søballe K, Hansen E, Brockstedt-Rasmussen H, Hjortdal V, Juhl G, Pedersen C, Hvid I, Bunger C (1991) Fixation of titanium and hydroxyapatite-coated implants in arthritic osteopenic bone. J Arthroplasty 6:307-316

Søballe K, Hansen E, Rasmussen H, Jorgensen P, Bunger C (1992) Tissue ingrowth into titanium and hydroxyapatite-coated implants during stable and unstable mechanical conditions. J Orthop Res 10(2):285-299

Stadlinger B, Pilling E, Huhle M, Mai R, Bierbaum S, Bernhardt R, Scharnweber D, Kuhlisch E, Hempel U, Eckelt U (2007) Influence of extracellular matrix coatings on implant stability and osseointegration: an animal study. J Biomed Mater Res B 83(1):222-231

Stadlinger B, Pilling E, Mai R, Bierbaum S, Berhardt R, Scharnweber D, Eckelt U (2008) Effect of biological implant surface coatings on bone formation, applying collagen, proteoglycans, glycosaminoglycans and growth factors. J Mater Sci 19(3):1043-1049

Stauber M, Müller R (2006) Age-related changes in trabecular bone microstructures: global and local morphometry. Osteoporos Int 17(4):616-626

Stauber M, Rapillard L, van Lenthe G, Zysset P, Müller R (2006) Importance of individual rods and plates in the assessment of bone quality and their contribution to bone stiffness. J Bone Miner Res 21(4):586-595

Szmukler-Moncler S, Salama H, Reingewirtz Y, Dubruille J (1998) Timing of loading and effect of micromotion on bone-dental implant interface: review of experimental literature. J Biomed Mater Res 43(2):192-203

Tsubota K, Adachi T, Tomita Y (2003) Effects of a fixation screw on trabecular structural changes in a vertebral body predicted by remodeling simulation. Ann Biomed Eng 31:733-740

Vajjhala S, Kraynik A, Gibson L (2000) A cellular solid model for modulus reduction due to resorption of trabeculae in bone. J Biomech Eng 122(5):511-515

van Lenthe G, Stauber M, Müller R (2006) Specimen-specific beam models for fast and accurate prediction of human trabecular bone mechanical properties. Bone 39(6):1182-1189 
Verhulp E, van Rietbergen B, Müller R, Huiskes R (2008) Indirect determination of trabecular bone effective tissue failure properties using micro-finite element simulations. J Biomech 41(7):1479_ 1485

Wermelin K, Suska F, Tengvall P, Thomsen P, Aspenberg P (2008) Stainless steel screws coated with bisphosphonates gave stronger fixation and more surrounding bone. Histomorphometry in rats. Bone 42(2):365-371

Wirth A, Mueller T, Vereecken W, Flaig C, Arbenz P, Müller R, van Lenthe G (2010a) Mechanical competence of bone-implant systems can accurately be determined by image-based micro-finite element analyses. Arch Appl Mech 80:513-525
Wirth A, Müller R, van Lenthe G (2010b) Computational analysis of small endosseous implants in osteoporotic bone. Eur Cells Mater 20:58-71

Yeh O, Keaveny T (1999) Biomechanical effects of intraspecimen variations in trabecular architecture: a three-dimensional finite element study. Bone 25(2):223-228

Zhang Q, Tan S, Chou S (2004) Investigation of fixation screw pull-out strength on human spine. J Biomech 37(4):479-485

Zhang Q, Tan S, Chou S (2006) Effects of bone materials on the screw pull-out strength in human spine. Med Eng Phys 28(8):795-801 